\title{
Changing Institutional Goals and Beliefs of Autonomous Agents
}

\author{
Guido Boella ${ }^{1}$ and Leendert van der Torre ${ }^{2}$ and Serena Villata ${ }^{1}$ \\ 1 Dipartimento di Informatica, University of Turin, Italy \\ 2 Computer Science and Communication, University of Luxembourg, Luxembourg
}

\begin{abstract}
Agents are autonomous and thus their goals and beliefs cannot be changed by other agents or the environment, but from outside the agents we can change their institutional goals and beliefs, that is, the responsibilities and powers associated with their roles. In this paper, we introduce a model of institutional dynamics, where the dynamics of an institution is modeled by the creation or removal of responsibilities and powers. We illustrate the change of institutional goals and beliefs using a government scenario.
\end{abstract}

\section{Introduction}

Institutions are structures and mechanisms of social order and cooperation governing the behavior of a set of individuals. However, the formal analysis of the institutions is challenging due to the complexity of its dynamics. For example, the institution itself may change over time due to the behavior of the agents. To model the dynamics of the institution, we use dependence networks developed by Sichman and Conte [11], as they are used in the early requirement analysis of Tropos [6].

The research question of this paper is: How to model the dynamics of dependence networks between agents due to institutional change? In our model presented in [3] we distinguish four views on multiagent systems without making institutions explicit. The agent view represents the agents of the systems introducing conditional goals, such as those goals that can be added by an agent to another one. The power view is presented as an abstraction of the agent view and it introduces two functions associating the agents with the goals they can create to agents and with the goals they can achieve. The dynamic dependence view is defined as an abstraction of the power view. In the model of [3], dependencies can be created by a set of agents making sets of agents dependent on each other, thanks to the power to trigger conditional goals. In this paper we present an extension of this model with also removal of dependencies. The challenge of the present paper is to explain how on the one hand agents are autonomous and, thus, their goals and beliefs cannot change, but on the other hand, the dependences between agents can change in an institution.

The paper is organized as follows. Section 2 introduces the running example, Section 3 and 4 introduce our model of institutions, and Section 5 introduces the dynamic social network. Related work and conclusions end the paper. 


\section{Running example: the Government Scenario}

The government scenario models the relations among ministers, where the prime minister has more powers than the other ministers, who have powers according to their domain. At the non-institutional level, a minister needs a ministerial car if she has to travel in town, she has to ask to the suitable office if she needs a translation service, she has to ask to the office of public relations to set a press conference if she needs to release a press statement, she has to contact the office with the job to update web site and to ask it to do the changes if she needs the publication on the web site of a particular office of new information, and so on. Each item can be modeled as a goal of the minister and the agents on which she depends to achieve her goals. For example, she depends on the office of public relations to achieve the goal to program a press conference. Together, these dependencies can be modeled as a social network.

From an institutional point of view, the government structure is hierarchical, in the sense that the Prime Minister has more powers that of all the other ministers, and the ministers have more powers than deputy-ministers. Consequently, the ministers depend on the prime minister. If the Prime Minister delegates to the foreign secretary some diplomatic topic, then all the other ministers and the secretaries have to refer to the foreign secretary regarding this topic. Likewise, the Prime Minister can also remove a delegated topic again, for example if there is a case of uncorrect behavior, taking her delegations ad interim. From this moment, it will be the Prime Minister to which the other ministers have to refer. Ministers also depend on each other. These institutional dependencies among ministers is typically given by authorizations. For example, the minister of transport needs an authorization from the minister of infrastructures to bridge a river, or the minister of public works needs funding from the minister of finance to call for tenders to build the bridge. These examples show how a minister M1 having a goal G1 depends on minister M2 to achieve it to have the authorization.

The institutional powers of the Prime Minister can create new dependencies among ministers. His institutional powers are that he can give a permission to the other ministers to do something, for example the permission to be absent to a council of ministers for serious reasons, and he can create obligations to other ministers, for example, the Prime Minister can oblige the minister of transports to present a document within a precise date. If the Prime Minister creates such an obligation, then he may create also a dependency of the minister to other ministers to obtain the document. Moreover, consider the example in which the minister of Transports depends on the minister of Infrastructure to have the authorization to start to build a new road. If the minister of Infrastructure is under investigation for corruption and then he is removed from his role of minister, then the Prime Minister can take the office of the minister of Infrastructure and so all his powers. In this case, the minister of Transports depends on the Prime Minister to obtain his authorization to start building the road. 


\section{Social Viewpoints for Dynamic Dependence Networks}

In our model introduced in [3], four views are defined to describe a multiagent system: the agent view, the power view, the dynamic dependence view and the coalition view. While the agent view represented the agents of the systems, their goals and the actions that they can perform to achieve these goals, the power view, based on the concept of social power introduced by Castelfranchi [8], introduced conditional goals, such as those goals that can be added by an agent to another one. We defined the dynamic dependence view as an abstraction of the power view, represented by a set of dependencies that can be added thanks to existence of conditional goals. Abstracting from the dynamic dependence view, we defined the coalition view representing a coalition as a set of dynamic dependencies where each agent either creates a dependency or fulfills a goal.

In this model, the power to trigger a goal is distinguished from the power to fulfill a goal. Due to the power to create goals, dependence relations are no longer static, but they can be created by agents. We extended the dependence networks developed by Conte and Sichman [11], following our previous results in [7]. In the abstraction from the power view to a dynamic dependence network, the creation of a dynamic dependency is based only on the power to create goals. In other models, creating a dependency can also be due to creation of new skills of agents. For formal details, see [3].

These four views can be applied to our running example since every minister has a set of private goals, beliefs and abilities and, according to the actions he can perform he has the power to see to a number of goals, his or of the other agents. Conditional goals are represented in our scenario, for example, by the decision of the Prime Minister to give to the minister of transports to present a document within a precise date. In this case, the Prime Minister has the power to trigger this particular goal. These conditional goals are the base of the change inside the dependence networks, transforming it in a dynamic dependence network.

\section{The Institutional View}

A social structure is modeled as a collection of agents, playing roles regulated by norms where "interactions are clearly identified and localized in the definition of the role itself" [13]. The notion of role is notable in many fields of Artificial Intelligence and, particularly, in multiagent systems where the role is viewed as an instance to be adjoined to the entities which play the role.

The institutional view is defined as follows:

Definition 1 (Institutional view (IV)). $I V=\langle R L, I F, R G, X$, igoals $: R L \rightarrow$ $2^{R G}$, iskills $: R L \rightarrow 2^{X}$, irules $\left.: 2^{X} \rightarrow 2^{I F}\right\rangle$ consists of a set of role instances $R L$, a set of institutional facts $I F$, a set of public goals attributed to roles, a set of actions $X$, a function igoals that relates with each role the set of public goals it is committed to, a function iskills that describes the actions each role can perform, and a set of institutional rules irules that relates a set of actions and the set of institutional facts they see to. 
The institutional view assigns to each participant a set of public goals, describing what he can do, e.g. authorize to built a bridge, and should do, e.g. be present at a council of ministers. Our scenario allows to enforce the behavior of the agents in the institution, for example, by blocking them from making statements contradicting facts, or by performing forbidden (virtual) actions, such as e.g. embezzle public money.

The social reality is provided with two distinct views, the material one, called the agent view in [3], and the institutional one that aims to regulate the behaviour of the agents. In a multiagent system each agent has a set of facts and goals that the other agents cannot change since agents are autonomous, formally presented in the agent view. Thanks to its existence inside a social structure, to each agent is added also new sets of facts and goals called the institutional ones and that can be viewed and also modified by the other agents as regards their institutional role.

The agents start with their sets of personal goals and beliefs and, only after their insertion inside a particular social structure they enlarge their sets of goals and beliefs. In particular, the set of goals is enlarged with new normative goals that represent the responsibilities of the agent inside its social structure while the set of beliefs is enlarged with new normative beliefs representing the set of constitutive norms of the systems, norms based on the collective acceptance of the society representable by means of an institutional ontology.

An Institutional Social Network is a social network representing set of individuals regulated by norms and containing the application of social roles to each individual involved.

\section{$5 \quad$ Institutional Dynamic Dependence Networks}

The passage from one institutional view to another one can be viewed as a dynamic social dependence network composed by all the social dependence networks coupled with the different institutional views. The main changes, that can occur to the institutional view to make it dynamic and pass from an institutional view to another one, are the addition or deletion of an igoal, of an iskill and of an irule. These additions and deletions change the number of dependencies and what agents are involved in them, passing from a social dependence network to another one. This change can be represented by means of dynamic social dependence networks. We extend the definition of dynamic dependence network proposed in [3] with the possibility not only to add dependencies between agents but also to remove them. Dynamic dependence networks are defined as follows:

Definition 2 (Dynamic Social Dependence Networks (DDN)). $A d y$ namic social dependence network is a tuple $\left\langle A, G\right.$, dyndep $^{-}$, dyndep $\left.{ }^{+}\right\rangle$where:

- $A$ is a set of agents and $G$ is a set of goals.

- dyndep ${ }^{-}: A \times 2^{A} \times 2^{A} \rightarrow 2^{2^{G}}$ is a function that relates with each triple of a agent and two sets of agents all the sets of goals in which the first depends on the second, unless the third deletes the dependency. The static dependencies are defined by $\operatorname{dep}(a, B)=\operatorname{dyndep}^{-}(a, B, \emptyset)$. 
- dyndep ${ }^{+}: A \times 2^{A} \times 2^{A} \rightarrow 2^{2^{G}}$ is a function that relates with each triple of a agent and two sets of agents all the sets of goals on which the first depends on the second, if the third creates the dependency.

The following definition introduces measures for existing dependencies and achieved goals. The overall value assigned to each dependence network is the ratio among the total number of goals that can be achieved by the agents of the network and the total number of dependencies among these agents.

\section{Definition 3 (Measures).}

- Number of goals that are achieved inside the network.

- nsg: $|S|$ where $S=\left\{G_{1}, G_{2}, \ldots, G_{n}\right\} \forall G_{i=1, \ldots, n} \in \operatorname{dep}\left(A_{1}, A_{2}, G_{i}\right)$

- Number of dependencies inside the network.

- $n d:|D|$ where $D=\left\{\operatorname{dep}_{1}, \operatorname{dep}_{2}, \ldots, \operatorname{dep}_{n}\right\} \forall d e p_{i=1, \ldots, n} \in D N=\left\langle A, G, \operatorname{dep}_{i}\right\rangle$.

- The goals-dependencies ratio is defined as:

- $G D-$ ratio $=\frac{n s g}{n d}$ with $n s g \leq n d$.

Since a dynamic social dependence network is a superimposition of a number of dependence networks, we define a $\Delta_{G D-\text { ratio }}$ that expresses how much changes the ratio between the number of achieved goals and the number of dependencies among the different dependence networks composing a dynamic dependence network.

Example 1 illustrates the addition of an irule and of an iskill by role instance Pm (Prime Minister). In the first case, the Prime Minister adds a new irule, that joins the institutional action $i x_{N}$ with the institutional goal $p g_{N}$, that it allows to achieve. Since the minister able to perform the institutional action $i x_{N}$ is the minister of transport (Tm) and the two ministers, infrastructure (Im) and finance $(\mathrm{Fm})$, have the institutional goal $p g_{N}$, there is the setting of two new dynamic dependencies. In the second case, the Prime Minister adds a new institutional iskill $i x_{e}$ to the minister of finance that allows the creation of a new dynamic dependence in which the Prime Minister depends on the minister of finance to achieve the institutional goal $p g_{5}$, allowed by $i x_{e}$.

Example 1. Consider the following additions:

- Role Pm adds to the institutional view a new irule: $I V \rightarrow I V+\{$ irules $U$ $\left.\left\{\left\{i x_{N}\right\},\left\{p g_{N}\right\}\right\}\right\}$ where $\left\{p g_{N}\right\} \subseteq$ igoals $(\operatorname{Im})$ and $\left\{p g_{N}\right\} \subseteq$ igoals $(F m)$ and $\left\{i x_{N}\right\} \subseteq \operatorname{iskills}(T m)$ then $\operatorname{dep}_{2}=\operatorname{dep}+\operatorname{dyndep}^{+}\left(\operatorname{Im}, T m, P m,\left\{p g_{N}\right\}\right)+$ $\operatorname{dyndep}^{+}\left(F m, T m, P m,\left\{p g_{N}\right\}\right)$ and $n s g_{2}=n s g_{1}+1$ and $n d_{2}=n d+2$;

- Role Pm adds to the institutional view also a new iskill: $I V \rightarrow I V+$ $\left\{\right.$ iskills $\left.\cup\left\{F m,\left\{i x_{e}\right\}\right\}\right\}$ where $\operatorname{irules}\left(K,\left\{p g_{5}\right\}\right)$ and $\operatorname{ability}\left(\left\{p g_{5}\right\}\right)=\left\{i x_{e}\right\}$ such that $\left\{p g_{5}\right\} \subseteq K$ and $\left\{p g_{5}\right\}=\subseteq$ igoals $(P m)$ then $\operatorname{dep}_{2}=\operatorname{dep}+\operatorname{dyndep}^{+}\left(\mathrm{Pm}, \mathrm{Fm}, \mathrm{Pm},\left\{p g_{5}\right\}\right)$ and $n s g_{2}=n s g+1$ and $n d_{2}=$ $n d+1$

Example 2 represents the application of the two additions on a dynamic social dependence network, as shown in Figure 1. 


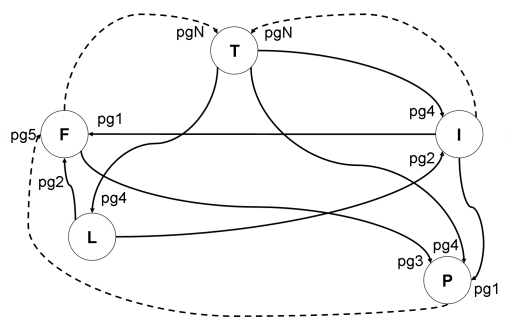

Fig. 1. DDN of Example 2 where dotted arcs represent new dependencies.

Example 2. Consider the following dynamic social dependence network:

1. Agents $A=\{T, I, F, L, P\}$ and Goals $G=\left\{p g_{1}, p g_{2}, p g_{3}, p g_{4}, p g_{5}, p g_{N}\right\}$;

2. $\quad-\operatorname{dep}(\{I\},\{P\})=\left\{\left\{p g_{1}\right\}\right\}$ : agent $I$ depends on agent $P$ to achieve goal $\left\{p g_{1}\right\}$

- $\operatorname{dep}(\{T\},\{I\})=\left\{\left\{p g_{4}\right\}\right\}$ : agent $T$ depends on agent $I$ to achieve goal $\left\{\mathrm{pg}_{4}\right\}$

- $\operatorname{dep}(\{L\},\{I, F\})=\left\{\left\{p g_{2}\right\}\right\}$ : agent $L$ depends on agents $I, F$ to achieve goal $\left\{p g_{2}\right\}$;

- $\operatorname{dep}(\{T\},\{P, L\})=\left\{\left\{p g_{4}\right\}\right\}$ : agent $T$ depends on agents $P, L$ to achieve goal $\left\{p g_{4}\right\}$;

- $\operatorname{dep}(\{I\},\{F\})=\left\{\left\{p g_{1}\right\}\right\}$ : agent $I$ depends on agent $F$ to achieve goal $\left\{p g_{1}\right\}$

- $\operatorname{dep}(\{F\},\{P\})=\left\{\left\{p g_{3}\right\}\right\}$ : agent $F$ depends on agent $P$ to achieve goal $\left\{p g_{3}\right\}$

- dyndep ${ }^{+}(\{I, F\},\{T\},\{P\})=\left\{\left\{p g_{N}\right\}\right\}$ : agents $I, F$ depend on agent $T$ to achieve goal $\left\{p g_{N}\right\}$ if it is created by agent $P$;

- dyndep ${ }^{+}(\{P\},\{F\},\{P\})=\left\{\left\{p g_{5}\right\}\right\}$ : agent $P$ depends on agent $F$ to achieve goal $\left\{p g_{5}\right\}$ if it is created by agent $P$;

3. The following measures show that, thanks to an increase of the number of dependencies, also the number of achieved goals increases: $G D-$ ratio $_{1}=\frac{4}{8}$ and $G D-$ ratio $_{2}=\frac{6}{11}$ with $n s g \leq n d ;$

\section{Related work}

Sierra [12] introduces Electronic Institutions (EIs) providing the virtual analogue of human organizations in which agents, playing different organizational roles, interact to accomplish individual and organizational goals. Roles are defined as patterns of behavior and the purpose of their normative rules is to affect the behavior of agents by imposing obligations or prohibitions. Another approach to EIs is given by [4]. They propose the use of 3D Virtual Worlds where an institution is represented as a building where the participants are represented as avatars and once they enter the building their actions are validated against the specified institutional rules. The problem of dynamic institutions is treated 
in [5] as an extension to EIs definition with the capability to decide in an autonomous way how to answer dynamically to changing circumstances through norm adaptation and changes in institutional agents.

As originally defined, dependence networks lack two ingredients: a normative structure and a dynamic representation of networks of social structures. Normative multiagent systems provide agents with abilities to automatically devise societies coordinating their behavior via obligations, norms and social laws [2]. The definition of power of Boella [1] can be directly applied to the description of the institutional view. Also the ability to achieve goals can be directly defined in terms of facts, skills and goals attributed to roles following the definition given in [1]. The presented formal model can be extended with obligations, as in [2]. Dependencies due to norms like obligations and permissions can be modeled by means of social dependence networks as in [7], however, institutional powers cannot be captured by the existing dependence networks formalism, since they introduce a dynamic element. By exercising a power, an agent transforms a social dependence structure into a new one by adding or removing dependencies at the institutional level of the social structure. Thus, in our paper, power is seen as the base of the change differently from what expresses by Jones and Sergot [10] and Grossi [9].

\section{Conclusions}

In this paper we show how to model the dynamics of dependence networks between agents due to institutional change, where the institutions are used to enforce the global behaviour of the society and to assure that the global goals of the society are met. Roughly, the behavior of the agents leads to a set of institutional facts, leading to the addition and removal of responsibilities and powers associated with the roles. This change of the institutional goals and beliefs of agents constitutes the dynamic behavior of the institution. The challenge of this paper is to explain how on the one hand, agents are autonomous and, thus, their goals and beliefs cannot change, but on the other hand, the dependences between agents can change in an institution. This challenge is twofold. First, we explain how we can change their institutional goals and beliefs, that is, the responsibilities and powers associated with their roles. Second, we explain how institutional goals are distinguished from the private goals and beliefs.

The change of institutional goals and beliefs of the agents is explained, first, by an informal example based on an hypothetical government. Second, it is explained by additions and deletions of dependencies between the agents using dependence networks developed by Sichman and Conte [11]. Moreover, we define measures to analyze dependence networks. As illustrated by the government scenario, the uniform combined model of role playing agents with private and institutional powers and goals provides an intuitive representation for dynamics in terms of modification of the institution, and the network measures are used to analyze this dynamics. 
The distinction between changing institutional goals and beliefs and changing private goals and beliefs is based on the notion of institutional power. We introduce the notion of institution based on the previously defined model of cognitive agent of [3], associating to each agent a role instance with its institutional goals, beliefs and capabilities. In [3] there is no definition of institution and the changes are governed by power and are represented by conditional goals, so in this paper we present an extension to the definition of dynamic dependence network of [3] with also removal of dependencies. Due to the concept of institutional power, the present paper shows the possibility to change the institutional goals and beliefs of the agents maintaining agents' autonomy.

\section{References}

1. Boella, G. and Sauro, L. and van der Torre, L., From social power to social importance, Web Intelligence and Agent Systems, IOS Press, pp. 393-404, (2007).

2. Boella, G. and van der Torre, L., Power in Norm Negotiation, Proceedings of KESAMSTA'0\%, LNCS, Springer, pp.436-446, (2007).

3. Boella, G. and van der Torre, L. and Villata, S., Social Viewpoints for Arguing about Coalitions, Proceedings of PRIMA '08, (2008).

4. Bogdanovych, A. and Esteva, M. and Simoff, S. and Sierra, C. and Berger, H., A Methodology for Developing multiagent Systems as 3D Electronic Institutions, Proceedings of AOSE@AAMAS'07, pp.358-360, (2007).

5. Bou, E. and Lopez-Sanchez M. and Rodriguez-Aguilar J. A., Adaptation of Automatic Electronic Institutions Through Norms and Institutional Agents, Engineering Societies in the Agents World VII, pp.300-319, (2007).

6. Bresciani, P. and Perini, A. and Giorgini, P. and Giunchiglia, F. and Mylopoulos, J., Tropos: An Agent-Oriented Software Development Methodology, Autonomous Agents and Multi-Agent Systems Journal, 8, pp.203-236, (2004).

7. Caire, P. and Villata, S. and van der Torre, L. and Boella, G., Conviviality Masks in Role-Based Institutions Multi-Agent Teleconferencing in Virtual Worlds, Proceedings of $A A M A S^{\prime} 08$, pp.1265-1268, (2008).

8. Castelfranchi, C., The micro-macro constitution of power, Protosociology, 18, pp. 208-269, (2003).

9. Grossi D., Designing Invisible Handcuffs: Formal Investigations in Institutions and Organizations for Multi-agent Systems, PhD Thesis, SIKS Dissertation Series 2007$16,(2007)$.

10. Jones, A. J. I. and Sergot, M., A Formal Characterization of Institutionalised Power, Logic Journal of IGPL, (2003).

11. Sichman, J. S. and Conte, R., Multi-agent dependence by dependence graphs, Proceedings of AAMAS'02, pp. 483-490, (2002).

12. Sierra, C. and Rodriguez-Aguilar, J. A. and Noriega, P. and Arcos, J. L. and Esteva, M., Engineering multi-agent systems as electronic institutions, European Journal for the Informatics Professional, 5, pp.33-39, (2004).

13. Zambonelli, F. and Jennings, N. and Wooldridge, M., Developing multiagent systems: The Gaia methodology, IEEE Transactions of Software Engineering and Methodology, 12, pp. 317-370, (2003). 